\title{
Resistencia a la terapia endocrina en cáncer de mama lobulillar
}

- Carlos Bonilla, Iván Pisciotti

Instituto Nacional de Cancerología, Bogotá, D.C.

Contacto: pisciotico@yahoo.es

Introducción y objetivos. El cáncer de mama es la principal causa de muerte entre las mujeres en el mundo, siendo el $75 \%$ receptores hormonales $(\mathrm{RH})$ positivos. La resistencia adquirida a la terapia endocrina resulta en progresión de la enfermedad.

Materiales y métodos. Femenina de 43 años con antecedente de ca lobulillar mama RH+ HER 2-, manejo inicial con quimioterapia AC, ooforectomía y mastectomía seguida por radioterapia (RT), libre de enfermedad por cuatro años, con posterior presencia de segundo primario versus metástasis, manejado con mastectomía con reporte patológico RH+ HER2- lobulillar, tratamiento adyuvante con docetaxel ciclofosfamida, seguido por RT y manejo hormonal con letrozol por dos años con posterior progresión ósea, iniciándose manejo con everolimus-exemestane.

Resultados. Resolución de síntomas óseos, sin efectos adversos adicionales por terapia con everolimus, con supervivencia libre de progresión por cuatro meses.

Conclusiones. La terapia con everolimus es una opción de tratamiento en cáncer de mama lobulillar resistente a la terapia endocrina.

\section{Carcinoma mucinoso puro de mama: experiencia en el Instituto Nacional de Cancerología entre 2006 a 2015}

\author{
- Kelman Hanael Ojeda Rodríguez, María Isabel Rizo, Jesús Oswaldo Sánchez, Sandra Díaz, Óscar Messa, \\ Sergio Mejía, David López
}

Instituto Nacional de Cancerología, Bogotá, D.C.

Contacto: namlek74@hotmail.com

Introducción y objetivos. El carcinoma mucinoso es un subtipo histológico del cáncer de mama, representando el $2 \%$ de casos. Se subdivide en puros (90\%) y mixtos. Presenta un mejor pronóstico con sobrevida global estimada a cinco años hasta en el $94 \%$. Según inmunohistoquímica, suelen expresar receptores hormonales y ser negativos para HER2, además manifiestan diferenciación neuroendocrina en el $15 \%$ al $50 \%$ de casos. En Colombia no se dispone de estudios al respecto. Objetivo: describir las características clínicas, epidemiológicas y respuesta clínica del carcinoma mucinoso puro de mama y el subgrupo con expresión neuroendocrina en el Instituto Nacional de Cancerología (INC) entre los años 2006 a 2015.

Materiales y métodos. Estudio descriptivo observacional, tipo serie de casos. Criterios de inclusión: >18 años, patología de carcinoma mucinoso puro. Fuente: registro de patología e historias clínicas. Análisis estadístico con Stata 11.0. Se estimará cálculo de proporciones en variables cualitativas, medidas de centralización y dispersión para las cuantitativas.

Resultados. Resultados preliminares. Reporte definitivo en 2016. De 1.181 casos de cáncer de mama, 30 pacientes $(2,54 \%)$ corresponden a carcinoma mucinoso puro. Edad promedio 65 años. Estadios: I $(6,7 \%)$, IIA (33\%), IIB (10\%), IIIA (6\%), IIIB (16\%\%) y IV (10\%). Sin compromiso nodal $(50 \%)$ y el $16 \%$ sin información. Luminal $A$ $(56,6 \%)$, luminal B $(30 \%)$, triple negativo $(6,67 \%)$, marcadores neuroendocrinos: $63,3 \%$ (sin información). Tratamiento en neadyuvancia $(13,2 \%)$, cirugía radical (40\%), cirugía conservadora (20\%), adyuvancia hormonal $(50 \%)$, quimioterapia adyuvante $(26,6 \%)$ y radioterapia adyuvante $(26,6 \%)$.

Conclusiones. Resultados compatibles con los publicados internacionalmente. Pendiente correlación con expresión de marcadores neuroendocrinos y comportamiento clínico. 